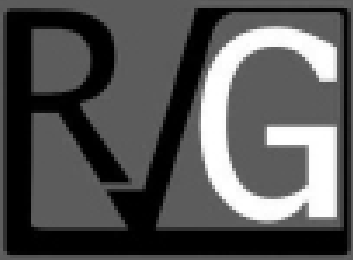

Año 24 No. 88

Octubre - Diciembre 2019

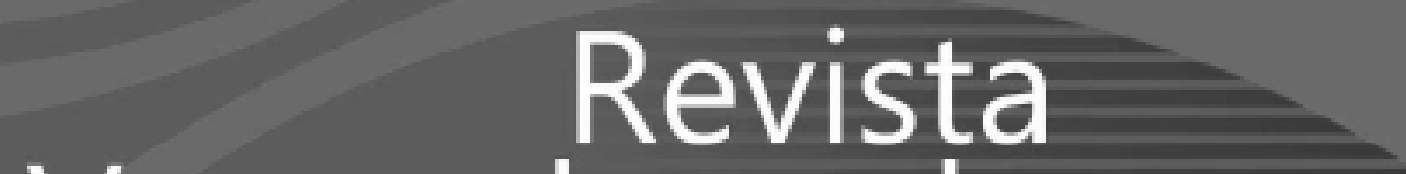

Venezolana de

verencla

UNIVERSIDAD DEL ZULIA (LUZ)

Facultad de Ciencias Económicas y Sociales

Centro de Estudios de la Empresa 


\title{
Capacidades dinámicas frente a la incertidumbre: una mirada desde la gestión universitaria ${ }^{1}$
}

\author{
Annía González, María Eugenia ${ }^{2}$ \\ Villalobos Antúnez, José Vicente ${ }^{3}$ \\ Ramírez Molina, Reynier Israel ${ }^{4}$ \\ Yanelis, Ramos Martínez ${ }^{5}$
}

\section{Resumen}

El estudio forma parte de una investigación realizada en la Universidad Privada Dr. Rafael Belloso Chacín, ubicada en Maracaibo, Venezuela; con la finalidad de analizar las capacidades dinámicas de la organización universitaria frente a la incertidumbre, con el diseño, técnicas y herramientas de la investigación cualitativa, desde el paradigma post positivista y con enfoque introspectivo vivencial, realizándose interpretación dialógica a las unidades emergentes surgidas de seis entrevistas en profundidad en las cuales intervinieron ocho sujetos participantes de la universidad. Se estableció como conclusión

\section{Recibido: 26-06-19 Aceptado: 17-09-19}

Artículo derivado de la Tesis Doctoral en Ciencias Gerenciales, titulada: "Formación de conciencia ética como proceso de transferencia de conocimiento en universidades privadas". Universidad Privada Dr. Rafael Belloso Chacín (URBE), 2018.

Universidad privada Dr. Rafael Belloso Chacín. Maracaibo. Venezuela. Directora de la Escuela de Derecho. Doctora en Ciencias Gerenciales. Co-Editora de la Revista Comertium et Tributum. Correo: merag2212@gmail.com

2 Profesor e Investigador Titular Medio Tiempo Internacional, del Departamento de Derecho y Ciencias Políticas de la Universidad de la Costa, Barranquilla-Atlántico, Colombia. Profesor e Investigador Titular de la Universidad del Zulia (LUZ), Venezuela, Coordinador del Diplomado en Bioética y del Laboratorio de Filosofía de la Ciencia, Facultad Experimental de Ciencias, Universidad del Zulia (LUZ).

3 Profesor invitado de las Universidades Pedro de Valdivia; Católica de Temuco, de Los Lagos (Chile); Benemérita Universidad Autónoma de Puebla, México; Católica de Bogotá y Colegio Mayor de Cundinamarca (Colombia). Correo: jvillalo4@cuc.edu.co; jvvillalobos@gmail.com

4 Profesor e Investigador Tiempo Completo, Departamento Ciencias Empresariales, Universidad de la Costa. Barranquilla, Colombia. Correo: rramirez13@cuc.edu.co

5 Investigadora de la Universidad Católica de Temuco, Chile. Profesora y tutora de tesis de Maestrías y Doctorado, Universidad Privada Dr. Rafael Belloso Chacín, Maracaibo, Venezuela. Árbitro internacional de revistas indizadas. Correo: yanelisramos6@gmail.com 
Capacidades dinámicas frente a la incertidumbre: una mirada desde la gestión universitaria

Annía González, María Eugenia; Villalobos Antúnez, José Vicente; Ramírez Molina, Reynier Israe y Yanelis, Ramos Martínez

que cuando la universidad privada está alerta y preparada para actuar efectivamente, se "blinda" ante la incertidumbre de su entorno; que aun cuando el ritmo de crecimiento se haya visto afectado para el momento del estudio, sus capacidades dinámicas en continua retroalimentación han sido el fundamento para mantenerse a la vanguardia en la oferta y demanda del servicio que presta, en la transferencia del conocimiento, su activa participación y fundamental rol que cumple como actor social en la sociedad venezolana, más allá de las fronteras del país.

Palabras clave: capacidades dinámicas; capacidades dinámicas universitarias; incertidumbre.

\title{
Dynamic capabilities in the face of uncertainty: a look from university management
}

\begin{abstract}
The study is part of a research conducted at the Dr. Rafael Belloso Chacín Private University, located in Maracaibo, Venezuela; with the purpose of analyzing the dynamic capacities of the university organization in the face of uncertainty, with the design, techniques and tools of qualitative research, from the post-positivist paradigm and experiential introspective approach, through the dialogic interpretation made to the emerging units arising from six in-depth interviews involving eight subjects participating in the private university. It was possible to discern by way of conclusion that when the private university is alert and prepared to act effectively, it "shields" itself from uncertainty as its environment; that even when the rhythm of its sustained growth has been affected, its dynamic capabilities in continuous feedback, have been the basis of staying at the forefront in the supply and demand of the service it provides, in the transfer of knowledge, its active participation and fundamental role that he plays as a social actor in Venezuelan society, beyond the country's borders.
\end{abstract}

Keywords: dynamic capabilities; dynamic university capabilities; uncertainty.

\section{Introducción}

La incertidumbre encierra multiplicidad de significados y sentidos desde el enfoque o perspectiva en la cual se quiera definir, toda vez que forma parte integrante de la vida humana. En la dinámica social, tanto individuos como grupos, se enfrentan a la vacilación, irresolución, perplejidad, indecisión, inseguridad, titubeo, fluctuación, dilema, imprecisión, duda, desconfianza, sospecha, escrúpulo, recelo, reparo, incredulidad, escepticismo, como sinónimos de la incertidumbre, cuando ella trastoca la estabilidad o "piso" 
donde se asientan los fundamentos a partir de los cuales fluyen y viven, particularmente, al afectar de manera negativa las cotidianas actividades que se desarrollan para la satisfacción de las necesidades primarias de subsistencia.

Históricamente, es en la incertidumbre generada por las "crisis sociales" producto de guerras, malos gobiernos, debacles económicas, hecatombes, desastres naturales, entre otros factores, donde individuos, grupos, organizaciones y sociedades, se han erigido exitosamente, mediante la creatividad e innovación, fortalecimiento de las mejores prácticas, conocimiento, valores, principios y el trabajo. Annía $(2018)^{5}$ y Annia, Villalobos, Romero, Ramírez \& Ramos (2018), expresan que cuando la universidad privada se enfrenta a factores políticos, económicos y sociales inestables, cambiantes, autoritarios y controladores de la vida social, que escapan de su propio control; los cuales van adecuando un marco jurídico legalizado, a veces no legitimado; cuyos efectos se perciben en el malestar social, inseguridad jurídica, inestabilidad económica, abusos de poder, entre otros; tal panorama lo puede enfocar de dos maneras: 1) como forma de autodestrucción, y 2) como horizonte de nuevas oportunidades.

En la primera, campea el desánimo, desmoralización, corrupción, miseria material y espiritual, de los individuos y de las organizaciones. En la segunda, se descubren la producción e innovación; se construyen nuevas formas de construir, crear y moldear, material y espiritualmente, individuos y grupos, personas y organizaciones, sociedad". En otras palabras, frente a tales amenazas del entorno que originan incertidumbre, la organización universitaria enfoca sus recursos, los cuales en palabras de Camacho \& Marcano (2003) y Assia (2007), se orientan con las capacidades de la organización. En efecto, los recursos de la organización pueden distinguirse entre tangibles e intangibles, siendo estos últimos los más considerados en los estudios referidos a las ventajas competitivas de las empresas.

Adicionalmente, para tener una ventaja competitiva sostenible potencial, los recursos de una empresa deben ser valiosos, raros, difíciles de imitar e insustituibles. Mientras que, la capacidad es la forma en que las empresas emplean sus recursos, se consideran un flujo o aspecto dinámico de las organizaciones. En tal sentido, el recurso es el conjunto de elementos y factores que, a través del conocimiento organizacional, como expresión de las capacidades, son conjugados por las empresas en estrategias para obtener ventajas competitivas, que las hagan efectivas, eficientes y rentables.

Ahora bien, si las capacidades están basadas en el conocimiento organizacional, no codificado, almacenado en la memoria organizativa que permite a las empresas actuar, al igual que los individuos de forma automática ante determinados estímulos; se pueden concebir como un conjunto de rutinas, que como pautas de actuación regulares

5 Annía G, María Eugenia. Formación de la Conciencia Ética como proceso de Transferencia del Conocimiento en Universidad Privada. Tesis Doctoral en Ciencias Gerenciales. Universidad Privada Dr. Rafael Belloso Chacín. Maracaibo. Venezuela. 2018. 
Annía González, María Eugenia; Villalobos Antúnez, José Vicente; Ramírez Molina, Reynier Israe y Yanelis, Ramos Martínez

y predecibles, indican cuáles son y cómo se realizan las tareas, por cuanto tales rutinas comprenden complejos patrones de interacción entre las personas y con los demás recursos, como resultado del aprendizaje colectivo de la organización acumulado lentamente en el tiempo, por lo que determinan lo que se puede hacer o se hace; todo lo cual implica el empleo de recursos en un limitado ámbito de posibilidades.

$\begin{array}{ccr}\text { Este conjunto de rutinas } \\ \text { organizativas } & 0 & \text { depósito del }\end{array}$ conocimiento representado en la forma como la empresa puede reproducir hábitos y actividades, siendo la innovación base de la experiencia y reproducción de las rutinas, es el vehículo a través del cual los directivos combinan variadas habilidades con experiencia funcional, para la creación de productos y servicios, así como construyen redes de colaboración entre las diferentes partes de la empresa, para crear combinaciones sinérgicas de recursos; adicionalmente, el proceso de transferencia, incluye rutinas de replicación y negociación, es utilizado por los directivos, para replicar, transferir y recombinar recursos, basado en el conocimiento dentro de la empresa.

De allí que, las capacidades dinámicas vienen a conformar una ampliación del significado de las anteriores definiciones, toda vez que estas son las facultades, potencialidades y posibilidades de las empresas para alimentar, adaptar y regenerar su base de conocimientos de forma consistente, para desarrollar y retener las capacidades organizativas que transforman esa base de conocimientos en acciones útiles. Al respecto, las habilidades integran, construyen y reconfiguran su dotación de recursos y capacidades, para responder al cambio del entorno.

Siendo entendido que la cualidad o especificación de "dinámicas" se refiere, precisamente a la habilidad de la organización, tanto para renovar capacidades como para alcanzar la congruencia con los entornos cambiantes, puesto que ciertas respuestas son requeridas cuando la tasa de cambio tecnológico es alta y cuando la naturaleza de los futuros competidores y de los mercados en el futuro son de difícil determinación.

Si los estudiosos han recomendado que, en entornos estables de una libre y sana competencia, las organizaciones deben orientar sus estrategias considerando necesidades y preferencias del mercado por igual a sus recursos y capacidades (Ramos, 2007); con más razón lo deben hacer cuando los entornos son turbulentos, en los que se producen cambios rápidos en la tecnología, así como en los gustos y necesidades de los consumidores, planteándose qué necesidades pueden satisfacer.

Pero, cuando los entornos son inestables, tal y como actualmente se desenvuelve la organización universitaria (Venezuela), se plantea cómo esta enfoca sus recursos y capacidades, particularmente, para el desarrollo de sus fines, funciones, objetivos y propósitos, donde el conocimiento: descubrimiento, creación, divulgación, publicación y transferencia, es lo principal. Es por ello, que este estudio analiza sus recursos y capacidades ante a la incertidumbre en la cual se desenvuelve.

Para realizar el estudio se toma como fundamento, los datos obtenidos y expuestos en un trabajo anterior ${ }^{6}$, mediante la selección de unidades 
emergentes que se desprenden del discurso de ocho informantes contenido en seis entrevistas en profundidad aplicadas, a través del empleo de diseño, técnicas e instrumentos propios de la investigación cualitativa, orientada por el enfoque epistemológico introspectivo vivencial, considerando que sus autores pasan de sujeto cognoscente a sujeto conocido, cuando forman parte del contexto, así como conviven en el espacio que comparten todos los sujetos, toda vez que interpretan y comprenden la realidad compartida de conciencia subjetiva, lo que hace que el enfoque sea introspectivo; así como también, sobre la experiencia investigativa precedente supra aludida.

De allí que este trabajo, como antes se expuso, toma como base los datos y resultados originales, recabados y obtenidos, en la interpretación dialógica de las seis entrevistas en profundidad realizada a ocho sujetos de la organización universitaria, contenidas en una previa investigación ${ }^{6}$. Siendo entendido que los indicados informantes hacen vida en la Universidad Privada Dr. Rafael Belloso Chacín (URBE), por lo que fueron seleccionados de conformidad a criterios correspondientes a su antigüedad o años de labor en la institución, y el cargo de tipo directivo.

A los fines de darle continuidad con el primigenio estudio fundamento de este trabajo, al igual que en dicha investigación, los investigadores autores se ubican en el sentido más amplio del paradigma post positivista como enfoque cualitativo para abordar la presente labor; esto es, donde la indagación, a partir de los supuestos, fue guiada por la teoría emergente que se desprendió de los hallazgos investigativos, validados mediante el diálogo, interacción y vivencia, para llegar a las conclusiones que se concretaron por medio de consensos generados por los procesos de observación, reflexión, diálogo, construcción de sentido compartido y sistematización.

En tal sentido, la investigación se orienta por el enfoque epistemológico introspectivo vivencial, considerando que sus autores pasan de sujeto cognoscente a sujeto conocido, cuando forman parte del contexto que delimita este estudio, así como conviven en el espacio que comparten con los sujetos conocidos, toda vez que interpretan y comprenden la realidad compartida de conciencia subjetiva, lo que hace que el enfoque sea introspectivo. Asimismo, dada la posición de los investigadores, se produce la simbiosis del conocimiento del sujeto - sujeto (objeto), puesto que se identifican, lo cual se constituye en una experiencia: vivida, sentida y compartida entre todos y por ello, la cualidad de vivencial del enfoque introspectivo.

Cabe destacar, que la URBE como organización y a su vez actor social dentro de la sociedad con la cual interactúa, comprende en su interior a un grupo heterogéneo de personas: estudiantes, docentes, personal obrero y administrativo; cuya conducta es el cúmulo de experiencias asumidas y hechas suyas, producto de las tradiciones, roles, valores y normas del ambiente, internalizadas paulatinamente, generando regularidades, guiando $\mathrm{y}$ determinando la conducta subsiguiente. De tal manera, que debe considerarse este proceso dinámico y la inclusión de todo aquel otro, que permite conocer y

7

Ob. Cit. (2) 
Annía González, María Eugenia; Villalobos Antúnez, José Vicente; Ramírez Molina, Reynier Israe y Yanelis, Ramos Martínez

reconocer la realidad de la organización universitaria como un sistema, de sus procesos y donde las partes que la componen, en su interactividad, establecen una relación de causa y efecto, entre las mismas partes.

Seguidamente, se toman unidades de análisis emergentes de seis entrevistas en profundidad, aplicadas a ocho sujetos participantes de la organización universitaria, previamente seleccionados conforme a determinados criterios, tales como: cualidad, capacidad, antigüedad y jerarquía en el cargo. La entrevistas realizadas en el desarrollo del trabajo ${ }^{8}$, fueron sometidas a la interpretación dialógica.

\section{La universidad privada y su entorno}

La universidad privada es una organización educativa autónoma, de libre ejercicio, sin injerencia de la nación, y sin financiación pública. Por lo tanto, los cambios políticos o presupuestos no les afecta considerablemente, su financiación proviene de sus propios ingresos, donaciones particulares o de patentes registradas por sus equipos de investigación. La organización universitaria objeto de estudio fue seleccionada originalmente en el trabajo primigenio de donde se extraen los datos para el estudio, entre otras razones, porque en palabras de Annía (2018) ${ }^{9}$ es la Universidad Privada Doctor Rafael Belloso Chacín (URBE) quien mejor describe la tercera misión de la universidad y el escenario propicio para la investigación, por cuanto se constituye en fuente inagotable de información referida a los propósitos perseguidos. En consecuencia, el proceso de transferencia del conocimiento deberá sustentarse por los valores, los principios y las políticas de la URBE, contenidos y guiados por la misión, para obtener y materializar la visión del futuro propuesto, en el espacio de la actuación universitaria dentro y fuera de la organización, de los individuos que participan y de la sociedad.

Así pues, se estima que la organización universitaria se desenvuelve en el término de la "tercera misión de la universidad", de acuerdo a la definición dada por Melero, Sanz y de Benito (2010) citados en Annía $(2018)^{10}$, las actividades vinculadas para la generación, aplicación, uso y explotación de la tecnología y el conocimiento de la universidad, fuera del entorno académico, mediante su relación con el resto de la sociedad; para lo cual, la universidad ha de adquirir responsabilidad y compromiso corporativo con la sociedad de la cual forma parte, especialmente, con su entorno inmediato: local y regional, aparte de generar y aplicar innovación.

Por lo tanto, debe motorizar la competitividad regional, a la vanguardia, impulsando el valor intelectual, tecnológico y científico, redireccionando la innovación hacia las auténticas necesidades que plantea la sociedad, orientada hacia el compromiso regional y la excelencia competitiva global, a través de una mejora continua del modelo innovador, emprendedor y científico, que la consagre como el eje vertebrador y de

\begin{tabular}{ll}
\hline 8 & Ob. Cit. (2) \\
9 & Ob. Cit. (2) \\
10 & Ob. Cit. (2)
\end{tabular}


referencia en su entorno.

En tal sentido, ubicada la universidad privada en la "tercera misión de la universidad", a ello se le suma su carácter de actor social, toda vez que según Annía (2018) los fines y funciones de esta, junto con los propósitos y objetivos que se plantea, claro que como sujeto parte de una sociedad globalizada, se han diversificado en dos sentidos: En primero lugar, por el descubrimiento y producción de conocimiento, a través de la investigación realizada pero también transferirlo, mediante la enseñanza, divulgación y publicación.

Segundo lugar, de acuerdo con lo planteado en el párrafo anterior, otro aspecto de la diversificación es la formación, capacitación y preparación de la persona en sentido integral, responsable, pero lo más importante, con alto sentido ético de su accionar para la conducción de los asuntos personales, profesionales, cívicos, ciudadanos y sociales que le involucran con su entorno. En tercer lugar, se manifiesta la diversificación mencionada por la integración al proceso de transferencia del conocimiento de las nuevas tecnologías y la innovación; esto último implica probar con otros métodos y mecanismos básicos e innovadores, de manera de poder producir conocimiento y divulgarlo, pero de forma responsable, esto es, con un alto sentido moral y ética. Por último, esta diversificación se aprecia en la idea de reconocerse y ser reconocida ella misma, esto es, la Universidad, como organización y sujeto transcendente, vinculante y vinculada con los procesos sociales, "de manera activa, voluntaria, participativa, protagónica y democrática; en la sociedad".

Cabe observar que, desde que se iniciara tal investigación ${ }^{11}$, la universidad privada ya se encuentra proactiva en el desarrollo de sus procesos, de tal manera disminuir las amenazas del turbulento entorno, donde la crisis social en Venezuela viene profundizándose; $y$, cuando se califica como social a la crisis, es porque este término engloba todos los factores y/o fenómenos que allí intervienen: políticos, económicos, culturales, tecnológicos y científicos; los cuales, por el contrario, en tiempos anteriores identificaban, tanto al país como a la sociedad venezolana, en proceso de crecimiento a la vanguardia del desarrollo de la ciencia y la tecnología, el cual hoy por hoy, se encuentra en total retraso.

Al respecto, arriba se expuso las formas en cómo puede la organización universitaria enfocar el panorama descrito, el cual se subsumea las primeras definiciones que en la introducción se hicieran sobre la incertidumbre. En otras palabras, que tal panorama lo puede enfocar de dos maneras: como 1) forma de autodestrucción, y 2) horizonte de nuevas oportunidades. En la primera, campea el desánimo, la desmoralización, corrupción, miseria material y espiritual, de los individuos y organizaciones. En la segunda, se descubre, produce y innova; se construyen nuevas formas de construir, crear y moldear, material y espiritualmente, individuos y grupos, personas y organizaciones, sociedad.

Además, deben sumarse las capacidades dinámicas de la organización universitaria, las 
Annía González, María Eugenia; Villalobos Antúnez, José Vicente; Ramírez Molina, Reynier Israe y Yanelis, Ramos Martínez

cuales destacan por su efectividad, excelencia y tiempo de respuesta, pese a las características propias de la incertidumbre de su entorno. Tales capacidades se reflejan en la inmediata transferencia interna del conocimiento en las rutinas de sus procesos: académicos, administrativos y mantenimiento; lo que le permite afrontar la adversidad: migración (estudiantil, docente y de personal), deserción y rotación, para la ejecución de actividades operativas y en desarrollo, como los procesos de inscripción, continuidad y culminación en tiempo del período académico, pese a paros de transporte, manifestaciones públicas, inflación económica y default. Adicionalmente y a la vanguardia, creación y puesta en marcha de "Urbe Internacional", por ejemplo, les permite a estudiantes que migran al extranjero, culminar sus carreras en Venezuela; a los docentes que migran, mantenerse vinculados con la institución.

En efecto, a saber de Annía (2018) ${ }^{12}$ las nuevas tecnologías orientadas a la transferencia han sido incorporadas por la organización universitaria, especialmente las más actualizadas, dada la vertiginosa velocidad en que estas se generan en el entorno en el cual se desenvuelve. Asimismo, la autora resaltó de la entrevista aplicada a los sujetos 1 y 2 de su investigación, información sobre los diversos vínculos que la universidad sostiene con múltiples y diferentes organizaciones del entorno venezolano, tales como instituciones financieras, farmacéuticas e instituciones de educación superior; mediante la aplicación tanto de estrategias como de herramientas tecnológicas e innovación, mismas de las nuevas tecnologías orientadas a la transferencia del conocimiento, lo que dio lugar al surgimiento de la universidad corporativa.

De igual modo, la referida autora señaló en su carácter de sujeto participante de la institución objeto de estudio, que esta ha ido más allá del entorno venezolano, en la implementación de tales estrategias y herramientas propias de las nuevas tecnologías orientadas a la transferencia del conocimiento, cuando además de la universidad corporativa, implementa y aplica tácticas e instrumentos en lo que denomina la universidad internacional, dedicada a la atención y difusión de programas de estudios de cuarto $y$ quinto nivel, dirigidos a extranjeros; expandiéndose fuera de las fronteras político territoriales, al abrir sus puertas esta casa de estudios en otro país: Estados Unidos de América.

También indicó, que con el aprovechamiento de las tecnologías, la institución desarrolla programas, tanto de la universidad internacional como la corporativa, en plataformas tecnológicas, para ser difundidos en sistemas automatizados y programas informáticos, como es el caso de blackboard, que permiten impartir contenidos y evaluación, en lo que se denomina educación a distancia, bien sea semi presencial o en línea (internet). La mencionada autora acotó que, debido a las amenazas del entorno de la organización, el ritmo de su sostenido crecimiento ha sido afectado. 


\section{El enfoque de los recursos en la gestión y transferencia del conocimiento}

Los recursos de la organización son todos los elementos que la conforman, la identifican y los usa, desarrolla, potencia en sus posibilidades, para su desenvolvimiento, Los autores les han atribuido múltiples significados de acuerdo a su naturaleza, fines, objetivos, características, entre otros; pero en lo que sí coinciden, es que estos son tangibles y/o intangibles, ubicando entre los intangibles, al conocimiento. Así pues, el conocimiento que caracteriza a toda organización en su forma de hacer las cosas o Know House, se transfiere internamente, puede externalizarse y depende de otro de sus recursos: los individuos que hacen vida en ella; pero, dicha transferencia no es total ni plena.

Los recursos por si solos no se orientan a ventajas competitivas, pues el empleo de estos exige la coordinación entre los recursos humanos, capacidad "conocimiento", activos fijos y recursos organizacionales (Boscán \& Villalobos, 2016; Hernández, Villalobos, Morales \& Moreno, 2016; Ramírez, Lay, Avendaño \& Herrera, 2018; Ramírez, Espindola, Ruíz \& Hugueth, 2019). Este conocimiento puede formar parte de aquel que es objeto y fundamento del servicio que presta, por ejemplo, una organización universitaria, es decir, del conocimiento científico, considerando, asimismo, los procesos que se llevan a cabo, sean previos, durante $y$ posteriores, a su producción y como objeto de su quehacer, para luego divulgarlo, publicitarlo y transferirlo, cumpliendo con ello, con sus objetivos, propósitos, fines y funciones. Siendo entendido que, cuando estos últimos se han diversificado, es porque se les ha incorporado la innovación y la tecnología, debido a la exigencia del entorno, lo que incluye constantemente transformar $y$ modernizar, en los métodos y medios de trasmisión del conocimiento, en búsqueda de las capacidades.

Por su complejidad, las capacidades son difíciles de categorizar y ubicar que los recursos, sin embargo, los recursos en la gestión son útiles para lograr un proceso sistemático dentro de la organización (Villalobos \& Bello, 2014; Villalobos \& Ganga, 2018; Rincón, Sukier, Contreras \& Ramírez, 2019; Villalobos, Guerrero \& Romero, 2019). De allí que, a saber de Annía (2018) ${ }^{13}$, las universidades, particularmente la universidad privada, al incorporar las nuevas tecnologías, la innovación y otros métodos para la transferencia del conocimiento y como parte de este proceso, se califica como organización de la tercera vía, toda vez que da cabida al desenvolvimiento de lo que se significa como tercera misión de la universidad.

En efecto, según la autora, entender, impulsar y transferir el desarrollo de la investigación y la innovación, son actividades que identifican la tercera misión universitaria, cuando estas se promueven fuera de su ámbito, entrando en este escenario la cualidad de interacción social de la transferencia del conocimiento, porque requiere de la participación de los diferentes actores sociales que 
Annía González, María Eugenia; Villalobos Antúnez, José Vicente; Ramírez Molina, Reynier Israe y Yanelis, Ramos Martínez

colaboran y se vinculan, tales como las empresas, el Estado, la sociedad; así como el aprovechamiento y distinción de los tipos de conocimiento: explícito y tácito, impulsando la inteligencia competitiva.

En este orden, la autora expone que, para garantizar el éxito de esta tercera misión de la universidad, es imponderable favorecer e impulsar la creación de redes estratégicas en las relaciones que se transformen en ventajas competitivas $y$ utilizar profundamente las nuevas tecnologías, mediante herramientas que fomenten positivamente la eficiente transferencia del conocimiento y su gestión.

Para ello, sostiene que se debe considerar la incidencia de ciertos factores, tales como los canales de vinculación, referidos a la frecuencia y profundidad bidireccional (doble sentido relacional) del contacto interpersonal; el grado de semejanza o acercamiento entre los sujetos involucrados; el detrimento, deterioro y/o pérdida que pudiere sufrir el conocimiento luego de la transferencia; el auto conocimiento de la organización, por parte de los sujetos que la componen y de ella misma como sujeto; la discordancia de los intereses y la congruencia de las metas individuales y organizacionales.

Por lo tanto, la transferencia del conocimiento se construye y constituye como fin y función de la universidad privada, toda vez que, además del descubrimiento y producción del conocimiento, a través de la investigación y transferirlo, mediante la enseñanza, divulgación y publicación; ello influye sustancial, sensible y decisivamente en la formación, capacitación y preparación de una persona integral, responsable, con alto sentido ético en la conducción de sus asuntos personales, profesionales, cívicos, ciudadanos y sociales.

En consecuencia, la autora expresa que la integración al proceso de transferencia del conocimiento de las nuevas tecnologías y la innovación, implica incursionar en otros métodos y mecanismos básicos e innovadores para producir conocimiento y divulgarlo, de manera responsable, moral y ética; todo lo cual conlleva a la universidad privada a reconocerse y ser reconocida como organización y sujeto, transcendente, vinculante y vinculada, de manera activa, voluntaria, participativa, protagónica y democrática, en la sociedad. Y que, de esta manera, la universidad privada, cumple con sus objetivos y propósitos, al establecer a la transferencia del conocimiento como fin y función.

En virtud de lo anterior, la organización universitaria enfoca sus recursos, los cuales como se expuso arriba, van aparejados con las capacidades, destacándose de estas últimas las dinámicas; seguidamente, resaltan al abordar la gestión del conocimiento en la universidad privada: la creación del conocimiento y el aprendizaje organizativo. Vale destacar, lo que a bien expone la teoría de los recursos y las capacidades, en cuanto a que la principal preocupación de las empresas para alcanzar el éxito, se relaciona con el uso eficiente de los recursos escasos, hecho que implícitamente está vinculado con la gestión del conocimiento y con el aprendizaje; por lo que se reitera lo expuesto anteriormente, sobre que para tener una ventaja competitiva sostenible potencial, los recursos de la organización deben ser valiosos, raros, difíciles de imitar e insustituibles. 
En efecto, a saber de Assia Gouza (2007) citado en Annía (2018) ${ }^{14}$, los recursos son valiosos, cuando permiten a las empresas concebir o implantar estrategias que aumenten su eficacia y eficiencia, así como generen ventajas competitivas de forma sostenible; son raros, cuando implanta una estrategia que crea valor y no es simultáneamente establecida por un número grande de empresas, puesto que lo contrario no le otorgaría ventajas competitivas; son insustituibles, cuando el recurso, que es fuente de ventaja competitiva sostenible, no debe de tener recursos valiosos (raros 0 imitables) estratégicamente equivalentes.

De igual modo, sostiene que los recursos son imperfectamente imitables, cuando reúne las siguientes condiciones: a) la habilidad de una empresa de obtener un recurso, depende de sus condiciones históricas únicas; b) el lazo entre los recursos que la empresa posee y la ventaja competitiva sostenible de la empresa, es causalmente ambiguo; y c) el recurso generador de la ventaja competitiva, es socialmente complejo. Ahora bien, con base a la ventaja competitiva, como una estrategia creadora de valor que no ha sido implementada por un competidor $y$, que si esta, además, es sostenible, ello implica que ningún competidor es capaz de duplicarla, aún teniendo la intención de hacerlo; por lo que la búsqueda de ventaja competitiva sostenible, debe centrase, en consecuencia, en la heterogeneidad y la inmovilidad de los recursos.

dobe conjugarse con la gestión
de conocimiento, la creación del conocimiento y el aprendizaje organizativo, como procesos inherentes al Enfoque de los Recursos que, igualmente, comprende las capacidades dinámicas. Al respecto, Assia Gouza (2007) citado en Annía (2018) ${ }^{15}$, expresa que tales procesos forman parte de enfoque de la gestión del conocimiento, el cual es producto del advenimiento de la economía del conocimiento y la sociedad de la información, que ubican a los recursos intangibles en la base de la ventaja competitiva de la empresa, siendo el conocimiento, el recurso intangible más estratégico, pero también el más complejo de gestionar, el cual constituye una ventaja competitiva a largo plazo, dada sus características de complejidad social, ambigüedad causal e idiosincrático, así como que se distingue de los recursos tradicionales de la empresa, lo cual requiere el diseño de nuevas estrategias, donde su gestión es la de flujos de conocimiento intra organizativo e inter organizativo.

Por su parte, la gestión de conocimiento es la que explica los procesos de creación y transferencia o muestra sus principales metas y objetivos, sin necesidad de definir lo que se entiende por el conocimiento, puesto que su labor es facilitar $y$ gestionar actividades relacionadas con el conocimiento, tales como la creación, captura, transformación y su uso, con el objeto de mejorar la capacidad de resolución de problemas y así contribuir a la sostenibilidad de las ventajas competitivas, en la empresa.

Además, dicha gestión del conocimiento va más allá de la propia de la tecnología o de la información, pero que siendo necesarias, no deben

\begin{tabular}{ll}
\hline 14 & Ob. Cit. (2) \\
15 & Ob. Cit. (2)
\end{tabular}


Annía González, María Eugenia; Villalobos Antúnez, José Vicente; Ramírez Molina, Reynier Israe y Yanelis, Ramos Martínez

ser el pilar fundamental sobre el que se sustenten los procesos de creación y transferencia del conocimiento; toda vez que el sistema de la información y de la comunicación, ha sido desarrollado para soportar la transferencia del conocimiento, así como que la práctica muestra que la tecnología no puede aumentar la distribución voluntaria del conocimiento por sí sola.

De las actividades de gestión de conocimiento, destacan la identificación, creación, desarrollo, transformación, renovación, difusión, aplicación o utilización del conocimiento, entre otras, por lo que la transferencia del conocimiento se ha transformado en uno de los procesos más críticos de la gestión del conocimiento. Así pues, la creación de conocimiento es un mecanismo individualizado que depende de la capacidad de cada persona y de sus experiencias de aprendizaje pasadas, por lo que una empresa no puede crear conocimiento sin la interacción de sus individuos, lo cual requiere de un entorno adecuado. En tal sentido, el nuevo conocimiento siempre se inicia en las personas y colocarlo a disposición de los demás, constituye la actividad fundamental de la empresa creadora de conocimiento.

De allí que, el proceso de la creación de conocimiento, supone necesariamente un contexto específico, en términos de quién participa y cómo participa, por lo que es necesario concentrar el conocimiento en un cierto tiempo y espacio. Sobre el aprendizaje organizativo, vale señalar que el aprendizaje es un concepto que se puede aplicar a las personas, los equipos y las organizaciones; por lo que el aprendizaje organizacional requiere de herramientas o mecanismos que permitan convertir al conocimiento de las personas y equipos de la empresa, en conocimiento colectivo, toda vez que se trata de una concepción que engloba los componentes cognitivos y de comportamiento, vinculada al conocimiento, según la cual el aprendizaje en la organización representa la transformación, tanto del modo de pensar las cosas - aspectos cognitivos-, como del modo de hacer las cosas - aspectos del comportamiento.

Asimismo, en un entorno que se caracteriza por la complejidad y el dinamismo, la relevancia del conocimiento reside, además de en su posesión, en las habilidades para desarrollarlo, transformarlo y utilizarlo, con el objeto de alcanzar un estado de ajuste ininterrumpido con ese entorno; siendo estas habilidades el resultado del aprendizaje que se desarrolla en el seno de la organización y, por ello, el aprendizaje puede y debe ser considerado como una capacidad organizativa crítica, para sostener, de forma permanente, la consistencia entre las exigencias del entorno y los activos de conocimiento; por lo tanto, para que el conocimiento organizativo sea una verdadera fuente de valor, debe ser captado e institucionalizado por el sistema organizativo, de manera tal que, posteriormente, sea recuperado y aplicado, en cualquier momento de tiempo y en un contexto estratégico cualquiera. En este orden, el conocimiento relaciona el conocimiento actual de la empresa con el desarrollo futuro de innovaciones, que cuentan con una dependencia temporal, puesto que los conocimientos y las capacidades esenciales de la empresa, constituyen la base, a partir de la cual se desarrollan nuevos productos y se incrementa el conocimiento de la organización.

Mientras que, existen diferentes niveles de aprendizaje en función 
del grado en el que se trate de alterar la memoria organizativa, siendo que concretamente las organizaciones pueden aprender por dos vías: (1) Desarrollando las competencias estratégicas de la empresa y servir como base para el lanzamiento de nuevos productos o servicios, a través de la experimentación continua; (2) Desarrollando la habilidad de renovar o revitalizar a la organización. Cabe resaltar, la importancia de reconocer que, los procesos de aprendizaje requeridos para la renovación de las competencias claves, son muy diferentes de los que resultan útiles para explotar las competencias existentes.

Así pues, a saber de Annía $(2018)^{16}$, los sujetos entrevistados coinciden en cuanto a que el enfoque de los recursos de la universidad como organización, así como sus capacidades, se encuentran orientados en el principal servicio que presta, como lo es la transferencia de conocimiento, para lo cual ha desarrollado sus capacidades paulatinamente, de forma innovadora, al punto de trascender, de marcar la pauta como referente a otras organizaciones similares, de distinguirse, ser rentable, poseer diversas y numerosas ventajas competitivas, ajustándose a las necesidades del entorno como internas, siendo esto último, la fuente de sus capacidades dinámicas.

En efecto, para los sujetos 1 y 2 , la organización es una "Burbuja donde las cosas funcionan". De igual modo, puede observarse ello en las unidades de análisis que se desprenden del discurso del sujeto 3 , referidas a las capacidades dinámicas y a los recursos propios de la universidad, al destacar que "La universidad como actor social, toma los insumos de los requerimientos de la sociedad, para diseñar el perfil del profesional"; de tal manera que, la organización se orienta o debe guiarse en: "Continuidad de formación familiar en la escuela, liceo y universidad"; "Formación de la conciencia ética en el aula; Modelación de la conducta ética desde el comportamiento ético con proyección social"; "Incorporación de la ética en los pensum de estudios de todas las carreras profesionales como cátedra"; "Supervisión interna (talento humano) y externa (desempeño de los egresados en las empresas) = medición transferencia de conocimiento"; "Disciplina como obligación en la formación"; "El desempeño docente = modelación de la conciencia ética del futuro profesional"; "Formación de la conciencia ética en la evaluación del proceso de investigación del participante en extensión y postgrado".

Asimismo, para el sujeto 4, la universidad enfoca sus recursos, cuyas capacidades son "dinámicas" al respecto, porque se alimenta de sus procesos (rutinas) internos como de las necesidades del entorno que considera necesarias satisfacer, orientándolas hábilmente, lo que la distingue de organizaciones similares, cuando señala: "Formación continua en extensión como medio de vinculación de ambos conceptos: transferencia del conocimiento - valor familia corporativa en los egresados"; "Alianzas estratégicas para establecer la vinculación".

"El servicio comunitario reflejo de la responsabilidad social corporativa 
Annía González, María Eugenia; Villalobos Antúnez, José Vicente; Ramírez Molina, Reynier Israe y Yanelis, Ramos Martínez

vincula la familia universitaria con la comunidad mediante la transferencia del conocimiento"; "El servicio comunitario universitario autosustentable en el asesoramiento de PyMES, otra forma de vinculación"; "Tres pilares de extensión que vinculan valor familia corporativa, transferencia del conocimiento $y$ sociedad: Centro de Transferencia Tecnológica (certificaciones CITGO), Responsabilidad Social Corporativa (Servicio Comunitario, entre otros) y Formación Continua"; "Transferencia de valores: puntualidad, responsabilidad, identidad corporativa, compromiso organizacional, conocimientos técnicos, a través de los egresados"; "Transferencia del conocimiento a otras empresas a través del liderazgo modelado en URBE como medio de vinculación"; “Desempeño profesional en otras instituciones $=$ modelación de conducta $=$ transferencia del conocimiento".

"Modelación de conducta y transferencia del conocimiento mediante desempeño profesional $=$ cambios positivos en otras instituciones"; "Valores individuales producto de formación familiar que permean por el desempeño en las organizaciones $=$ transferencia del conocimiento"; "La competencia humana de la conciencia ética humana permite adaptación, flexibilización y generación de procesos de innovación, transformación, cambio además de su promoción"; "Cátedra libre, dinámica por su dimensión, eje transversal en todos los programas de formación = desarrollo de la competencia humana de conciencia humana, en los programas de pregrado y postgrado universitarios como oportunidad".

Luego, el sujeto 5 expresa la manera en que se orientan y enfocan los recursos y capacidades de la universidad, señalando como hábilmente dinamiza las últimas, en razón a las necesidades del entorno que pudiera satisfacer, generando ventajas competitivas a su favor, a saber: "Formación de profesional integral = recalcar valores de excelencia: responsabilidad, compromiso, puntualidad, imagen", "Somos abiertos para reforzar y complementar currículos"; "Se invita a la constancia, a cumplir compromiso: individual, organizacional y social"; "Creación de expectativas desde el ingreso"; "Clave del éxito: modelo de programas de actualización de competencias"; "URBE certifica competencias en el ámbito internacional, mediante Person View, CISCO y la rúbrica reconocida de sus autoridades, entre otros"; "Implementación de manual organizacional que comprende misión, visión y valores; en lugar visible"; "Transferencia de valores a través de salutación del máximo líder de la organización"; "Mensaje de modelación en valores: imagen, puntualidad, identificación con la organización, orientación, seguridad"; "Extensión comprende CTT (Centro de Transferencia Tecnológica) importante por participantes jóvenes, Diplomado para estudiantes último año de carrera y externos, Responsabilidad Social Universitaria (servicio comunitario)"; "Decanato de extensión = parte estratégica".

De las unidades de análisis que se desprenden del discurso del sujeto 7 , se evidencia lo que sería el "deber ser", al efecto, para el destinario que, a su vez, servirá de medio o vía de transferencia del conocimiento; mientras que, el sujeto 8 refiere que la universidad debe enfocar sus recursos y capacidades dinámicas en humanizarse, en virtud a los cambios políticos, sociales y económicos del entorno, cuando sostiene la importante 
necesidad de cátedra abierta en formación de valores, la necesidad del estudiante como función de la universidad y la profundización de la institucionalidad mediante la humanización.

\section{Conclusión}

De acuerdo al análisis de la variable de estudio frente a la incertidumbre, se puede extraer que:

a) son las capacidades dinámicas de la organización universitaria, claves para la obtención de destrezas y habilidades, las cuales deben evolucionar para desarrollar ventajas competitivas, articuladas con las necesidades del entorno, lo que le permite afrontar la incertidumbre; puesto que enfoca sus recursos, propios de la tercera misión de la universidad, en el cumplimiento, desarrollo y éxito de sus objetivos, propósitos, fines y funciones.

b) El hecho de encontrarse la universidad privada, en avance en relación a otras instituciones similares de su entorno; a la vanguardia en la creación y puesta en marcha de procesos, proyectos y planes, científicos y tecnológicos, para sí misma y transferirlos; permiten proyectar en el futuro "casi" inmediato, mismo de los intempestivos cambios que caracterizan a la incertidumbre como entorno organizacional, cuáles rutinas organizativas (depósito del conocimiento representado en la forma como la empresa puede reproducir hábitos y actividades, siendo la innovación base de la experiencia y reproducción de las rutinas; y, por otro lado, que a través de las rutinas, los directivos combinan sus variadas habilidades con experiencia funcional, para la creación de productos y servicios, así como construyen redes de colaboración entre las diferentes partes de la organización, para crear combinaciones sinérgicas de recursos) se aplican en un momento determinado, estudiados como fueran los posibles escenarios. Es decir, mantenerse alerta y preparada para actuar efectivamente, le permite "blindarse" ante la incertidumbre como su entorno.

c) Puede que el ritmo de su sostenido crecimiento se haya visto afectado; sin embargo, sus capacidades dinámicas en continua retroalimentación han sido el fundamento de mantenerse a la vanguardia en la oferta y demanda del servicio que presta, como lo es la educación superior, en la transferencia del conocimiento, en su activa participación y fundamental rol que cumple como actor social en la sociedad venezolana, más allá de las fronteras del país. Efectivamente, es una "burbuja" donde las cosas funcionan, dentro del contexto de incertidumbre en el cual se desenvuelve.

\section{Referencias bibliográficas}

Annía González, María Eugenia (2018), Formación de la Conciencia Ética como proceso de Transferencia del Conocimiento en Universidad Privada. Tesis Doctoral en Ciencias Gerenciales. Universidad Privada Dr. Rafael Belloso Chacín. Maracaibo, Venezuela.

Annía G., María Eugenia; Villalobos Antúnez, José Vicente; Romero Pabón, Julio César; Ramírez Molina, Reynier Israel \& Ramos Martínez, Yanelis (2018), Conciencia ética en organizaciones universitarias: un estudio fenomenológico de la raxis como continua formación. En Revista Inclusiones, Vol. 6 (4), 179-195. Santiago de Chile.

Assia Gouza (2007), Los factores de- 
Capacidades dinámicas frente a la incertidumbre: una mirada desde la gestión universitaria

Annía González, María Eugenia; Villalobos Antúnez, José Vicente; Ramírez Molina, Reynier Israe y Yanelis, Ramos Martínez

terminantes de la transferencia del conocimiento dentro de las spinoffs universitarias. Tesis doctoral. Universidad Autónoma de Barcelona. Bellatera, España.

Boscán Leal, José Antonio \& Villalobos Antúnez, José Vicente (2016), Bioética desde una perspectiva latinoamericana. En Argumentos de Razón Técnica, 19(1), 59-83.

Camacho, Hermelinda \& Marcano, Noraida. (2003). El enfoque de investigación Introspectiva vivencial y sus secuencias operativas. Algunos casos de estudio. En Revista Omnia, 9(1),1-21. Universidad del Zulia. Maracaibo, Venezuela.

Hernández, P., Villalobos, J., Morales, M., \& Moreno, J. (2016). Racionalismo emergente en la gerencia universitaria: factor de humanización en universidades de Colombia, Venezuela y México. En Revista Espacios, 37(30), 1-22.

Ramírez Molina, Reynier Israel; Espindola, Cesar; Ruíz, Gladis \& Hugueth, Alfredo. (2019). Gestión del Talento Humano: Análisis desde el Enfoque Estratégico. En Información Tecnológica,30(6), La Serena, Chile.

Ramírez Molina, Reynier Israel; Lay, Nelson; Avendaño, Inirida \& Herrera, Belina. (2018). Liderazgo resiliente como competencia potenciadora del talento humano. En Opción. Revista de Ciencias Humanas y Sociales, 34(86), 826-854. Maracaibo Venezuela.
Ramos Martínez, Yanelis. (2007). Estrategias de la gestión directiva y construcción de la grandeza docente en las Escuelas Bolivarianas. En REDHECS. Revista de Humanidades, Educación y Ciencias Sociales. Año 2 (2): 75-89. Maracaibo, Venezuela.

Rincón, Yanyn; Sukier, Harold; Contreras, Jairo \& Ramírez Molina, Reynier Israel. (2019). Responsible Communication Strategies for Small and Medium-Sized Enterprises. En Opción. Revista de Ciencias Humanas y Sociales, 35(89), 1208-1234. Universidad del Zulia. Maracaibo, Venezuela.

Villalobos Antúnez, José Vicente \& Bello, M. (2014). Ética para una sociedad global: la bioética puente para el giro tecnocientífico. En Revista Lasallista de investigación, 11(1), 70-77. Colombia.

Villalobos Antúnez, José Vicente \& Ganga Contreras, Francisco. (2018). Tecnoempresa y Tecnocimiento: Una Perspectiva desde la Bioética Empresarial. En Revista Fronteiras, 7(3), 214-230. Centro Universitario de Anapolis, Brasil.

Villalobos Antúnez, José Vicente., Guerrero, José., \& Romero, Luis. (2019). Hermenéutica de la política y legitimidad de su ejercicio: democracia y Estado de derecho. En Utopía y Praxis Latinoamericana, 24(86), 182-197, Universidad del Zulia. Maracaibo Venezuela.

\footnotetext{
- Esta obra está bajo una licencia de Creative Commons Reconocimiento-NoComercial- Compartirlgual 3.0 Unported. http://creativecommons.org/licenses/by-nc-sa/3.0/deed.es_ES
} 